\title{
Ureaplasma SPP and mycoplasma hominis in women of reproductive age with pelvic inflammatory disease
}

\author{
Dina Nuradilova ${ }^{1}$, Lira Kaliyeva ${ }^{1}$, Daiva Vaitkiene ${ }^{2}$, Saltanat Kalimoldayeva ${ }^{3}$, Khasen Dasibekov ${ }^{4}$, \\ Zbigniew Omiotek $^{5}$, Teresa Małecka-Massalska ${ }^{6}$
}

(1) Asfendiyarov Kazakh National Medical University, Department of obstetrics and gynecology No. 2, Almaty, Kazakhstan

(2) Lithuanian University of Health Sciences, Department of obstetrics and gynecology, Kaunas, Lithuania

(3) State-owned public enterprise with right of economic jurisdiction "Regional diagnostics center", Clinical Diagnostics Laboratory, Almaty, Kazakhstan

(4) Regional clinical hospital, Almaty, Kazakhstan

(5) Lublin University of Technology, Faculty of Electrical Engineering and Computer Science, Lublin, Poland

(6) Medical University of Lublin, Department of Human Physiology, Lublin, Poland

Date submitted:

Oct 05, 2018

Date accepted:

Oct 17, 2018

Online publication date:

March 15, 2019

\section{Corresponding Author: \\ Zbigniew Omiotek \\ Lublin University of Technology, \\ Faculty of Electrical Engineering and Computer Science, Lublin, \\ Poland \\ z.omiotek@pollub.pl}

Keywords: Pelvic inflammatory disease, Ureaplasma SPP, Mycoplasma hominis, infertility, pregnancy pathology, antibiotic sensitivity.

\section{ABSTRACT}

Aims:To determine the prevalence of infections with species Ureaplasma species (SPP) and Mycoplasma hominis and the antibiotic sensitivity in women of reproductive age with inflammatory diseases.

Methods:2360 samples were investigated, obtained by urethral and cervical canal scrapings of reproductive age women with pelvic inflammatory disease in the clinical diagnostic laboratory of the Regional diagnostic center of Almaty. The cultivation, identification and susceptibility testing of urogenital mycoplasmas and ureaplasmas to 9 antibiotics were conducted with the use of commercial kits.

Results:Among 2360 tested samples total infection (isolated USPP, Mycoplasma hominis and mixed infection) was high $(47.6 \%)$. The infection rate of Ureaplasma SPP was more than 30 times higher than the prevalence of Mycoplasma hominis $(23.0 \%$ and $0.7 \%$, respectively). Mixed infection was found in $23.8 \%$ of cases. Total infection of Ureaplasma SPP and Mycoplasma hominis was the highest in women aged $30-39$ years $(60.0 \%)$, less in women in the age group of $40-45$ years (31.4\%). Most active against Ureaplasma SPP and Mycoplasma hominis were tetracyclines. Indeed $94.1 \%$ of the isolated strains of Ureaplasma SPP and $98.2 \%$ of Mycoplasma hominis were sensitive to tetracycline. The doxycycline susceptibility of Ureaplasma SPP and Mycoplasma hominis was the highest (99.5\% and $98.3 \%$, respectively).

Conclusions:A high level of pefloxacin sensitivity allows to recommend this antibiotic to prescribe for women with pelvic inflammatory disease. In light of wide spread of strains of Ureaplasma SPP and Mycoplasma hominis, resistant to ofloxacin and macrolides, we do not suggest these antibiotics to treat the infections.

\section{Introduction}

Pelvic inflammatory disease (PID) does not only deteriorate the quality of women's life, but also reduces their fertility function. Currently many researchers believe the main etiological factor of PID are the urogenital infections, among which Ureaplasma SPP (USPP) and Mycoplasma hominis (MH) play a key role. According to many researchers, urogenital Mycoplasma and Ureaplasma are associated with inflammatory diseases of the genitourinary tract including pyelonephritis, cystitis, urolithiasis, and the risk of miscarriage, chorioamnionitis, postpartum and abortion fever (1-3).

Researchers have noted higher prevalence of Ureaplasma SPP than Mycoplasma hominis among the population (4-6). The role of Ureaplasma and Mycoplasma in the development of infertility is not fully understood. It is known that the USPP was detected more often than $\mathrm{MH}$ in the scrapings from the cervix of infertile women (7-9). According to most researchers, two infections by Chlamydia trachomatis and Ureaplasma SPP are directly associated with infertility $(9,10)$.

The widespread use in the practice of polymerase chain re- action (PCR) method has enhanced the ability of most laboratories to diagnose of ureaplasmosis and mycoplasmosis infections. But in some cases, when the infection continues to recur, despite the treatment, there is a need of determination of the sensitivity of microorganisms to antibiotics. Ureaplasma is extremely easily transmitted sexually and vertically from mother to fetus. The probability of this transmission reaches $90 \%$ (11). They are capable of adhesion to various cell types such as urethral epithelial cells, spermatozoa and erythrocytes $(2,12)$. Mycoplasma is an atypical bacterium that does not have its own cell walls such as Ureaplasma. Mycoplasma hominis normally colonises the urogenital tract asymptomatically but it can provoke postpartum and postoperative infections of the genitourinary and respiratory systems (13).

Contamination during pregnancy may lead to chorioamniotic pregnancy complications and neonatal infection (14). In many cases USPP and $\mathrm{MH}$ are not diagnosed due to the lack of symptoms, the antibacterial effect of semen and cultivation difficulties $(3,9)$. Urogenital mycoplasmas and ureaplasmas were isolated in a special class of Mollicutes due to their unique properties that distinguish them from most bacteria. These 
properties are the following: very small sizes, close to the size of viruses, lack of a rigid cell wall, polymorphism of cells (15). The most frequently USPP and $\mathrm{MH}$ are identified in genitalia and they grow well on nutrient medium. Therefore, in order to diagnose them, in most cases the culture method is used. It allows not only estimation of the number of microorganisms in the material under study, but also their susceptibility to antibiotics. It is considered that concentration of 104 microbes in one gram of a sample has diagnostic value. Lower concentration can be defined in healthy people $(16,17)$. Urogenital mycoplasmas and ureaplasmas are passed by sexual contact and passage of the fetus through the infected genital tract of the mother. The mycoplasmas' growth is suppressed by tetracyclines, fluoroquinolones and macrolides. Mycoplasmas and ureaplasmas are resistant to antibiotics that suppress the synthesis of cell wall components (penicillins, rifampicines) (16). In this regard, we undertook testing with the use of commercial kits for the cultivation, identification and susceptibility of urogenital mycoplasmas and ureaplasmas to antimicrobial agents. The aim of the study was to determine the prevalence and antimicrobial sensitivity of Ureaplasma SPP and Mycoplasma hominis in reproductive age women with pelvic inflammatory disease.

\section{Methods}

The research work has passed a local ethical examination at Asfendiyarov KazNMU, research protocols \#179 from $04 / 29 / 2015$ and $\# 345$ of $04 / 05 / 2016$, as the conclusion on approval by the local ethical commission is valid for one year.

Under the conditions of diagnostic laboratory of the Regional Diagnostic Centre of Almaty, 2360 samples (one sample from each woman) of biological material were investigated, obtained by scraping from the cervical canal and urethra of reproductive-aged women (from 17 to 45 years) with pelvic inflammatory disease. For clinical case of PID, isolated disease of the upper sections of the reproductive tract (cervicitis, endometritis, salpingitis, pyosalpinx, tubo ovarian abscess pelvic peritonitis) or any combination of them was taken.

Cultivation. The kit for cultivation, identification and susceptibility of USPP and $\mathrm{MH}$ to antibiotics is an 18-well system containing dried biochemical substrates and antibiotics. Commercial kits Mycoplasma System Plus are produced by the company Liofilchem, Italy and are available on the market. The system provides a semiquantitative assessment of the presence of urogenital mycoplasmas and ureaplasmas.

Identification. Biomaterial was placed in a special nutrient medium Mycoplasma Transport Medium immediately delivered to the laboratory. Next, the cooled samples were examined within 48 hours. Semi-quantitative counting of the urogenital USPP and $\mathrm{MH}$ was provided by a colour change from yellow to red in the wells: 1-GR+ (growth of the microorganism in the titer from 102 to $104 \mathrm{CFU} / \mathrm{ml}) ; 2-\mathrm{GR}++(104<$ titre<105 CFU/ml); 3-GR+++ (titre>105 CFU/ml). The presence of Mycoplasma hominis was confirmed using arginine test and the presence of Ureaplasma SPP - with a urea test. Mycoplasma metabolises arginine from the culture medium during the growth. In these conditions, the colour of the 4-ADC well varies from yellow to red. Ureaplasmas at cultivation consume urea, and colour of the small 5-UR well turns red from yellow.

Study of susceptibility to antibiotics. System of microorganisms' sensitivity determination to antibiotics consists of 9 antibiotics in two concentrations (tetracycline $4 \mathrm{mg} / \mathrm{l}$ and $8 \mathrm{mg} / \mathrm{l}$, pefloxacin $8 \mathrm{mg} / \mathrm{l}$ and $16 \mathrm{mg} / \mathrm{l}$, ofloxacin $1 \mathrm{mg} / \mathrm{l}$ and $4 \mathrm{mg} / \mathrm{l}$, doxycycline $4 \mathrm{mg} / \mathrm{l}$ and $8 \mathrm{mg} / \mathrm{l}$, erythromycin $8 \mathrm{mg} / \mathrm{l}$ and $16 \mathrm{mg} / \mathrm{l}$, clarithromycin $8 \mathrm{mg} / \mathrm{l}$ and $16 \mathrm{mg} / \mathrm{l}$, minocycline $4 \mathrm{mg} / \mathrm{l}$ and 8 $\mathrm{mg} / \mathrm{l}$, clindamycin $4 \mathrm{mg} / \mathrm{l}$ and $8 \mathrm{mg} / \mathrm{l}$; azithromycin $4 \mathrm{mg} / \mathrm{l}$ and $8 \mathrm{mg} / \mathrm{l})$. Sensitivity and resistance of microorganisms were assessed at three levels: $\mathrm{S}$ - sensitive (colour of the well is yellow); I - intermediate-sensitive (colour of the well - orange) and R-resistant (colour of the well - red). Before the procedure for taking the biomaterial, informed consent was obtained in advance from all the women surveyed. Statistical analyses were performed by using the statistical software package SPSS version 15 for Windows (SPSS Inc., Chicago, IL, USA). P-value of less than 0,05 was considered to indicate a statistically significant difference. A P-value of less than 0.05 was considered to indicate a statistically significant difference and all statistical calculations and analyses were performed using the statistical software package SPSS (version 15; Statistical Package for the Social Sciences, Chicago, USA).

\section{Results}

All the samples of biomaterial were examined for the presence of Ureaplasma SPP, Mycoplasma hominis and mixed infection. In case of detection of these infections, the sensitivity of microorganisms to antibiotics was conducted. Ureaplasma SPP was detected in 543 of 2360 examined women, that is $23.0 \%$ of the total number of patients. Mycoplasma hominis was identified less frequently - in $0.7 \%$ of cases (17 women). The mixed infection of Ureaplasma SPP + Mycoplasma hominis was detected in 563 women, i.e. $23.8 \%$ of the total number of examined patients. In general, 47.6\% (1123 samples) of women tested showed the presence of infection. The level of USPP infections was significantly higher than the level of $\mathrm{MH}$ infection (23.0\% and $0.7 \%$ respectively). The mixed infection prevalence $(23.8 \%)$ practically did not differ from the prevalence of isolated USPP (23.0\%).

The analysis of infection prevalence in various age groups of women with PID is shown in Table 1 . The highest overall infection rate was observed in the age group 30-39 $(60.0 \%)$, which is significantly higher than in other age groups $(P<0.05)$, whereby USPP in women of 30-39 years of age was found in

Table 1. Prevalence of Ureaplasma SPP and Mycoplasma hominis among women with pelvic inflammatory disease.

\begin{tabular}{c|ccccc}
\hline \multirow{2}{*}{$\begin{array}{c}\text { Ages of the } \\
\text { examined }\end{array}$} & U.SPP+ & M. hominis+ & Mixed infection & Positive & Negative \\
\cline { 2 - 6 } & $10(23.2 \%)$ & $0(0.0 \%)$ & $11(25.6 \%)$ & $21(48.8 \%)$ & $22(51.2 \%)$ \\
\hline under 20 & $306(24.6 \%)$ & $10(0.8 \%)$ & $162(13.0 \%)$ & $478(38.4 \%)$ & $767(61.6 \%)$ \\
\hline $20-29$ & $210(20.8 \%)$ & $7(0.7 \%)$ & $386(38.5 \%)$ & $603(60.0 \%)$ & $402(40.0 \%)$ \\
\hline $30-39$ & $17(25.4 \%)$ & $0(0.0 \%)$ & $4(6.0 \%)$ & $21(31.4 \%)$ & $46(68.6 \%)$ \\
\hline $40-45$ & $543(23.0 \%)$ & $17(0.7 \%)$ & $563(23.8 \%)$ & $1123(47.6 \%)$ & $1237(52.4 \%)$ \\
\hline Total & & & & &
\end{tabular}


$20.8 \%$ of cases; $\mathrm{MH}-$ in $0.7 \%$. Mixed infection was the most frequently identified in this age group (38.5\%). The prevalence of USPP, $\mathrm{MH}$ and mixed infections among women under 20 and in $20-29$ age group was $48.8 \%$ and $38.4 \%$, respectively.

The level of USPP infection among women younger than 20 $(23.2 \%)$ and in the age group 20-29 (24.6\%) was slightly different. $\mathrm{MH}$ was not found among women under 20 . Mixed infection was much more common in the age group up to $20(25.6 \%)$ than in women aged 20 to $29(13.0 \%)(P<0.05)$. However, a small number of samples in the age group up to 20 (43) compared to women of the age group from 20 to 29 (1245) does not allow to make firm conclusions. The age group of women from 40 to 45 years old was also small ( 67 people). The mixed infection rate in this group was $6.0 \%$ and was the lowest in comparison with other age groups $(P<0.01)$.

Isolated USPP was found in $25.4 \%$ of the samples. $\mathrm{MH}$ was not demonstrated among 40-45-year-old women. The research results of the infectious agents' susceptibility to antibiotics are presented in Table 2. The greatest antimicrobial activity was demonstrated by antibiotics from the tetracycline group. Thus, the Ureaplasma in $94.1 \%$ and Mycoplasma in $98.2 \%$ of women showed susceptibility to tetracycline. In mixed infection, antimicrobial susceptibility to tetracycline was confirmed in $89.2 \%$ of women. Susceptibility to doxycycline was even higher (USPP in $99.5 \%, \mathrm{MH}$ in $98.3 \%$, in case of mixed infection in $90.6 \%$ ). The level of susceptibility of microbes to minocycline was slightly lower (87.5\%, $78.4 \%$ and $68.1 \%$ respectively).

The antimicrobial susceptibility of agents to fluoroquinolones (ofloxacin, pefloxacin) was significantly lower, especially in the case of mixed infection. More than half of the mixed-infection strains were insensitive to ofloxacin (51.8\%). However, susceptibility of isolated mycoplasmas, ureaplasmas and mixed infections to macrolides was the lowest. Thus, half of the USPP strains appeared to be insensitive to clarithromycin and $59.4 \%$ of USPP samples were resistant to clindamycin. Sensitivity of ureaplasmas to erythromycin (61.6\%) and azithromycin $(63.3 \%)$ was slightly higher. Susceptibility of urogenital mycoplasmas to macrolides was minimal - to clarithromycin in $38.4 \%$, clindamycin in $27.9 \%$, to erythromycin in $24.3 \%$, and to azithromycin in $15.6 \%$ of the examined women. The strains with full resistance to all 9 antibiotics ( 5 women, i.e. $0.2 \%$ of the total number of examined) gives cause for concern.

\section{Discussion}

At present, the pathogenic role of mycoplasmas and ureaplasmas has not been entirely clarified. But when there are clinical manifestations of the inflammatory process in the genitourinary organs, impaired fertility, miscarriages, premature births, innidiation of newborns, antibiotics (tetracyclines, fluoroquinolones, macrolides) are prescribed. According to various authors, 40 to $80 \%$ of clinically healthy women are carriers of USPP (17-19). The results of our study showed the presence of isolated USPP in $23.0 \%$ of women of reproductive age with PID and $47.6 \%$ of women with mixed infection (USPP+MH). It showed highest percentage among women under 20 (48.8\%) and in women of $30-39$ years of age (60.0\%). Mixed infection (USPP $+\mathrm{MH}$ ) was also diagnosed in this group $(38.4 \%$ of the examined women). Isolated USPP infection was found out approximately at the same frequency in different age groups. Some researchers report the same prevalence in women of USPP and $\mathrm{MH}$ irrespective of their region of residence $(5,9,10)$. In the women surveyed, there was a clear predominance of USPP (23.0\%). MH was identified only in $0.7 \%$ of the examined women. Rodrigues R. et al. found that $47.6 \%$ of women with infertility had pathogens of urogenital infections, including $12.9 \%$ of Chlamydia trachomatis, $23.5 \%$ of Ureaplasma SPP, $4.8 \%$ of Mycoplasma hominis, and Chlamydia trachomatis and USPP were directly related to infertility (20).

The study of antibiotic susceptibility showed that most strains of USPP and $\mathrm{MH}$ are susceptible to three antibiotics of the tetracycline series (doxycycline, tetracycline, minocycline) and to one antibiotic from the group of fluoroquinolones - pefloxacin. According to the results of our research pefloxacin is preferable to prescribe in USPP or MH infection. In case of mixed infection, the third of the strains was resistant to it. Using macrolides in treatment of USPP and $\mathrm{MH}$ is inexpedient; this concerns especially $\mathrm{MH}$ and mixed infection. About $90 \%$ of samples containing mixed infection appeared to be resistant to macrolides. Thus, despite a wide arsenal of Ureaplasma SPP and Mycoplasma hominis laboratory identification methods (cultural, antigen identification, immunofluorescence test, polymerase chain reaction, antibody detection), we consider that cultivation of mycoplasmas and ureaplasmas with determination of sensitivity to antibiotics is very important in an optimum antibiotic therapy.

Data on the prevalence of urogenital mycoplasmas and

Table 2. The antibiotic susceptibility of Ureaplasma SPP and Mycoplasma hominis (isolated and mixed infection) in women with pelvic inflammatory disease.

\begin{tabular}{|cccc|}
\hline Antibiotics & U. SPP (\%) & M. hominis (\%) & Mixed infection (\%) \\
\hline Tetracycline & 94.1 & 98.2 & 89.2 \\
\hline Doxycycline & 99.5 & 98.3 & 90.6 \\
\hline Minocycline & 87.5 & 78.4 & 68.1 \\
\hline Ofloxacin & 69.8 & 74.6 & 48.2 \\
\hline Pefloxacin & 83.1 & 90.2 & 67.1 \\
\hline Erythromycin & 61.6 & 24.3 & 10.6 \\
\hline Clarithromycin & 50.1 & 38.4 & 27.5 \\
\hline Clindamycin & 40.6 & 27.9 & 16.0 \\
\hline Azithromycin & 63.3 & 15.6 & 12.8 \\
\hline
\end{tabular}


ureaplasma are contradictory. Most authors indicate a higher prevalence among the population Ureaplasma SPP than Mycoplasma hominis $(4-6,14,17)$. Thus, according to various authors, from 40 to $80 \%$ of clinically healthy women of reproductive age are carriers of USPP (16-19). At the same time, some researchers point to an approximately equal level of infection of women USPP and $\mathrm{MH}$, regardless of the region of residence $(9,10,20)$. In contrast, the results of our study demonstrate a multiple (more than 30 times) excess infection of USPP women with PID than $\mathrm{MH}$. According to Russian authors, in the proportion of samples positive for urogenital infections received from women with PID, the proportion of USPP was $54.6 \%$, while $\mathrm{MH}$ $-5.1 \%$. Consequently, the excess was not so significant (more than 10 times) (21). The proportion of women with vaginal colonisation of $\mathrm{MH}$ increases after puberty and depends on the number of sexual partners. This was demonstrated in a study in which the genital colonisation of $\mathrm{MH}$ was detected in only 1 out of 91 women who had no sexual activity. At the same time, $\mathrm{MH}$ was identified in 15 out of 97 women with more than four sexual partners (22).

Until now, the role of USPP and $\mathrm{MH}$ in the etiology of infertility has not been fully clarified. Many authors believe that Chlamydia trachomatis and USPP are directly involved in the case of subclinical infection and tubal peritoneal infertility (7-8). According to Witkin et al. (21), it is USPP, not MH, that is found in the cervical scrapes of most women with infertility. Until now it has not been possible to statistically confirm the relationship between $\mathrm{MH}$ and infertility. But infection with $\mathrm{MH}$ during pregnancy can lead to chorioamniotitis, complications of pregnancy and to neonatal infection (3). Studies of the sensitivity of USPP and $\mathrm{MH}$ to antibiotics are not so numerous. Individual researchers note a combination of high sensitivity of USPP and $\mathrm{MH}$ to doxycycline and complete resistance to tetracycline (23).

\section{Conclusions}

The results of our study of the antibiotic susceptibility of USPP and $\mathrm{MH}$ indicate high efficacy of both tetracycline and doxycycline, but sensitivity to doxycycline was more frequent (in the case of isolated USPP and $\mathrm{MH}, 99.5 \%$ and $98.3 \%$, respectively; in cases of mixed infection - $90.6 \%$ ). As the most active antibiotics against USPP and $\mathrm{MH}$, some authors call doxycycline from among the antibiotics of the tetracycline series and josamycin from macrolides. But recently, most researchers note a high proportion of strains of USPP and $\mathrm{MH}$, resistant to macrolides (josamycin, clarithromycin and erythromycin). The results of our studies showed that the most active against USPP and $\mathrm{MH}$ were tetracyclines and pefloxacin. A high proportion is noted of USPP and MH resistant to ofloxacin and macrolides.

\section{Acknowledgments}

DN directed the study, collected the data and contributed the writing of the manuscript. LK designed the study. DV analyzed the study results and contributed the revision of manuscript. SK performed microbiological research and contributed the writing of the manuscript. KD collected the data. ZO performed the statistical analysis of the data. TM-M reviewed the study results.

The research was conducted at the expense of the Health Department of Almaty in the framework of the innovative project "Optimization of laboratory diagnostics of urogenital infections in women of reproductive age with pelvic inflammatory diseases."

\section{Conflict of interest}

On behalf of all authors, the corresponding author states that there is no conflict of interest.

\section{References}

1. Imudia AN, Denti L, Puscheck EE, et al. The prevalence of Ureaplasma urealyticum, Mycoplasma hominis, Chlamydia trachomatis and Neisseria gonorrhoeae infections and the rubella status of patients undergoing an initial infertility evaluation. J Assist Report Genet. 2008;25(1):4346.

2. Waites KB, Katz B, Schelonka RZ. Mycoplasmas and ureaplasmas as neonatal pathogens. ClinMicrobiol Rev. 2005;18(4):757-789.

3. Mutrha AP, Edwards JM. The role of Mycoplasma and Ureaplasma in adverse pregnancy outcomes. Obstetrics and gynecology clinics of North America. 2014;41(4):615-627.

4. Sobouti B, Fallah S, Mobayen M, et al. Colonization of Mycoplasma hominis and Ureaplasmaurealyticum in pregnant women and their transmission to offspring. Iran J microbial. 2014;6(4):219-224.

5. Zdrodowska-Stefanov B, Klosowska W, Ostarzewska-Ruchalska I, et al. Ureaplasma urealyticum and Mycoplasma hominis infections in women with urogenital diseases. Adv Med Sci. 2006;51:250-253.

6. Vertesamo R, Patella A, Calzolari E, et al. An epidemiological survey of Mycoplasma hominis and Ureaplasma urealyticum in gynecological outpatients, Rome, Italy. Epidemiol Infect. 2013;141(12):2650-2657.

7. Pellati D, Mylonakis I, Bertoloni G, et al. Genital tract infection and infertility. Eur J Obstet Gynecol Report Biol. 2008;140(1):3-11.

8. Gdoura R, Kchaou W, Ammar-Keskes L, et al. Assessment of Chlamydia trachomatis, Ureaplasmaurealyticum, Ureaplasma partum, Mycoplasma hominis, and Mycoplasma genitalium in semen and first void urine specimens of asymptomatic male partners of infertile couples. J Androl. 2008;29(2):198-206.

9. Michou IV, Constantoulakis P, Makarounis K, et al. Molecular investigation of menstrual tissue for the presence of Chlamydia trachomatis, Ureaplasma urealyticum and Mycoplasma hominis collected by women with a history of infertility. J Obstet Gynecol Res. 2014;40(1):237-242.

10. Gû́nueli I, Abike F, Dúnder I, et al. Chlamydia, Mycoplasma and Ureaplasma infections in infertile couples and effects of these infections on fertility. Ach Gynecol Obstet. 2011;283(2):379-385.

11. Miron ND, Socolov D, Mares M, et al. Bacteriological agents which play a role in the development of infertility. Acta Microbiol Immunol Hung. 2013;60(1):41-53.

12. Kong F, Ma Z, James G, et al. Molecular genotyping of human Ureaplasma species based on multiple-banded antigen (MBA) gene sequences. Int J Syst Evol Microbiol. 2000;50(5):1921-1929.

13. Xiao L, Glass JI, Paralanov V. Detection and characterization of human Ureaplasma species and serovars by real-time PCR. J Clin Microbiol. 2010;48(8):2715-2723.

14. Miranda C, Camacho E, Reina G, et al. Isolation of My- 
coplasma hominis from extra genital cultures. Eur J Clin Microbiol Infect Dis. 2005;24(5):334-337.

15. Gilbert GL. Chlamydial and mycoplasmal infection. Seminar Neonatol. 1996;1:119-126.

16. Ullman U, Schubert S, Krause K. Comparative in vitro activity of levofloxacin other fluorquinolones, doxycyclin and erythromycin against Ureaplasmaurealyticum and Mycoplasma hominis. J Antimicrob Chemother. 1999;43(C):33-36.

17. Daxboeck F, Iro E, Tamussino K, et al. Bacteremia with Mycoplasma hominis and Ureaplasma urealyticum in patients undergoing hysterectomy. Eur J Clin Mycrobiol Infect Dis. 2003;22(10):608-611.

18. Keane FE, Thomas BJ, Gilroy CB, et al. The association of Mycoplasma hominis, Ureaplasma Urealyticum and Mycoplasma genitalium with bacterial vaginosis: observations on heterosexual women and their male partners. Int J STD AIDS. 2000;11(6):356-360.

19. Baka S, Kouskouni E, Antonopoulou S, et al. Prevalence of Ureaplasma urealyticum and Mycoplasma hominis in women with chronic urinary symptoms. Urology. 2009;74(1):62-66.

20. Rodriguez R, Hernandez R, Fuster F, et al. Genital infection and infertility. Enferm Infecc Microbiol Clin. 2001;19(6):261-266.

21. Witkin SS, Kligman I, Grifo JA, et al. Ureaplasma urealyticum and Mycoplasma hominis detected by the polymerase chain reaction in the cervices of women undergoing in vitro fertilization: prevalence and consequences. J Assist Reprod Genet. 1995;12(9):610-614.

22. Paavonen J, Miettinen A, Stevens C, et al. Mycoplasma hominis in nonspecific vaginitis. Sex Transm Dis. 1982;10(4):271-275.

23. Dégrange $\mathrm{S}$, Renaudin $\mathrm{H}$, Charron $\mathrm{A}$, et al. Tetracycline resistance in Ureaplasma spp. and Mycoplasma hominis prevalence in Bordeaux, France from 1999 to 2002 and description of two tet(M)-positive isolates of Mycoplasma hominis susceptible to tetracyclines. Antimicrobial Agents and Chemoterapie. 2008;52(2):742-744. 